\title{
Quest for Other Worlds Awakens
}

\author{
Jean Schneider from the Observatoire de Paris describes current programmes and \\ long-term plans at a time when interest is on the increase following recent \\ announcements of some intriguing results.
}

The problem of the existence or not of other worlds has today two, heterogeneous aspects. We would like to answer several astrophysical questions (frequency of the occurrence of other planetary systems, similarity to the solar system, formation, evolution, dependence on major features of the central star). But there also are far more important questions which relate to the evolution of man: "Is there life on extrasolar planets?"; "Are we alone in the Universe?". The standard view is that life is supported by complex, out-of-equilibrium chemical processes so it requires specific conditions which are only fulfilled by what are defined as "habitable" planets. Several decades of discussion have, perhaps provisionally, brought us to the conclusion that a habitable planet has a temperature of $\approx 300 \mathrm{~K}$ (to allow for liquid water).

In addressing the question of the existence of other worlds one is therefore prompted to adopt a clearly defined four-fold strategy involving searches for any kind of extrasolar planets, for habitable planets, for signs of life on these planets, and for alien "intelligence".

As far as the three first are concerned, much work has started in the USA with a coherent national programme originally called TOPS (Towards Other Planetary Systems) but now referred to as ASEPS (Astronomical Studies of Extrasolar Planetary Systems). The contrast with Europe, where the field is underdeveloped, is striking although it may, hopefully, change in the near future.

\section{Two Types of Planets}

The potential success of a detection method clearly depends not only on technological limitations but also on the characteristics of the planets being sought (their mass $M$, radius $R$, temperature $T$, and distance $a$ from the parent star). There are regions in the $M-R-T-a$ domain which are likely to be forbidden because planets are either solid or gaseous. It is an empirical fact that all the solid planets or planetary cores in the solar system have a mass at most 15-times the Earth mass $M_{\odot}$. In the absence of any other information, one takes the conservative view that while a gaseous planet can have a Jupiter size and mass (or greater), a solid extrasolar planet has at most 15 Earth masses or $\approx 2.5$ Earth radii.

On the other hand, a gaseous (Jupiterlike) planet, defined as having a temperature below $200 \mathrm{~K}$ to prevent hydrogen evaporation, must be at least 5 Astronomical
Units (AU) from the parent star. This constraint leads to two categories of planets:

- massive gaseous planets $\left(M \geq 15 M_{\odot}\right)$ which are at the same time outer planets in a sequence;

- Earth-like solid planets $\left(M \leq 15 M_{\odot}\right)$ which are at the same time inner planets.

If one accepts that a habitable planet has a temperature of $300 \mathrm{~K}$, only inner planets, being fairly close to the central star, can develop some kind of biochemical activity.

\section{Several Viable Approaches}

A planet reveals itself either by its own emission or by some kind of perturbation in the star's characteristics. The insert reviews some of the observed effects, the phases of most of which are modulated owing to the orbital revolution of the planet. The magnitudes of the effects are summarized in the Table, where the numbers in the two central columns are for a parent star with 1 solar mass at $5 \mathrm{pc}$ from the Sun (for other planetary systems, these numbers scale in an obvious way with the planet and star parameters). They must be compared with the

\section{Experimental Approaches} planet. planet. incompatible with a large radio-emission from the planet. present or future performance of astronomical instrumentation.

The Table leads to obvious conclusions which deserve comment. The most common methods, namely imaging, astrometry and accelerometry, are in fact sensitive only to the presence of outer planets (as far as current searches and soon-to-be-completed projects are concerned). There are naturally ambitious projects to detect Earth-like inner planets with these methods, but they have no chance of becoming operational for at least two decades.

As for the approaches valid for all planets, the most efficient is the timing method. Unfortunately, it only works for pulsars and is thus useless for "normal" (i.e., main sequence) stars. The gravitational amplification method has the disadvantages that it reveals the presence of a planet only once (during the single transit brought about by the proper motion of its parent star) and by itself does not allow for subsequent observations of a detected planet. However, in extensive surveys this method could provide statistical information on the number of planetary systems.

The occultation method is the only one which can detect, in the near future, inner planets around main sequence stars and give some of their characteristics. It could

A variety of phenomena are used in the search for Earth-like planets:

- The planet is revealed by reflection of the star's light on the planetary surface, with a difference in magnitude (log of the brightness) depending on the configuration of the system. - Since the planet is heated by the star, one can detect its excess infrared emission peaking at some wavelength depending again of the geometry of the system.

- During an orbital revolution of the planet, the star has a "reflex" motion around the common centre-of-mass of the star/planet system which leads to a variation in angular position of the star in the sky. This astrometric method exploits either optical or radio wavelengths. - Similarly, the gravitational perturbation generated by the planet gives a periodic modulation in the radial velocity of the star, with the same period as the orbital revolution of the

- If the star can be considered as a clock, as for a pulsar, its reflex motion owing to the presence of the planet modulates the time of arrival of the clock signals. Pulsars are generally considered as hostile environments for life. However, this approach deserves further investigation, because, for instance, lethal radio, $\mathrm{X}$ - and gamma-ray radiations may be damped by a sufficiently thick atmosphere as long as the pulsar beam does not hit the

- If the orbital plane of the planet is suitably oriented, it produces an occultation - a decrease in the star's light during transits (duration $\approx 1-20$ hours). For a random orientation of the planet's orbital plane, the geometric probability of the transit is $=1 \%$ for a single star. For an eclipsing binary, this probability can amount to $100 \%$ since the binary and planet orbital planes are identical (there is a double transit in this case).

- The planet can produce a gravitational amplification of the light from background stars, with a duration depending on the planet's transverse velocity.

- Finally the planet may have, like Jupiter and Saturn, an intrinsic radio emission which can be searched for. Based on present knowledge, this approach should work for any kind of planet. For instance, in the solar system, the Earth is brighter than Uranus and Neptune at decametric radio wavelengths. However, it is not clear whether the presence of life is 
perhaps also be used to probe, using absorption of the star's light, the planet's atmospheric composition.

\section{Some Important Results}

There are many projects and programmes aimed at detecting extrasolar planets. Most are under development, but some have already started to produce important results.

- Astrometry: an optical interferometer to measure perturbations in the positions of several hundreds of stars is being tested at Mount Palomar in the USA. Astrometric studies can also be made at radiowavelengths and a collaboration between the Observatory of Paris and the US Jet Propulsion Lab is currently using very long baseline interferometry to seek perturbations in the positions of some radio stars. The Hubble Space Telescope has monitored several stars for a numer of years with a precision of 1 marcsec. Benedict et al. announced in 1993 the possible discovery of a 0.8 Jupiter mass planet orbiting at $0.2 \mathrm{AU}$ from Proxima Cent (the nearest star at 3.5 light years). If this is confirmed, it would constitute the first discovery of a planet around a normal star.

- Spectroscopy: Several groups in Canada and the USA started some 12 years ago to monitor spectroscopically several tens of bright, nearby stars. In a spectroscopic survey of 21 solar-type stars, Walker et al. reported in 1994 that another planet with about twice the mass of Jupiter does not exist (at a significance level of three standard deviations). Although negative, this result is astrophysically very important. It is the first time we have an answer to the 2300 year old question: "Are there everywhere (i.e., around all other stars) planetary systems like our's?". The response is now clearly "no" since less than one star in 20 has Jupiters. We can already conclude that either planetary systems are infrequent or the G-stars (i.e., like the sun) in the solar neighbourhood do have planetary companions, but they are not like our's (for in spite of the absence of Jupiters at $a \leq 10 \mathrm{AU}$, they may well have inner Earth-like planets). The second possibility strongly encourages us to accelerate efforts to search for Earth-like planets around other main sequence stars.

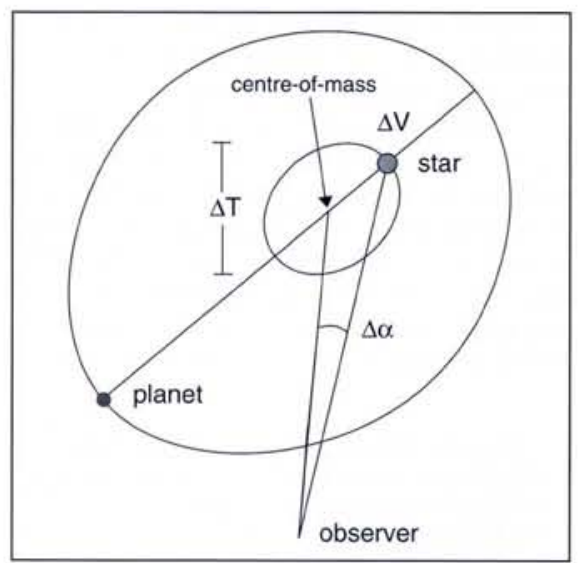

Some of the parameters that can be observed for a star perturbed by the presence of a planet. Indicated are the position wobbling $\Delta \alpha$; velocity perturbation $\Delta V$; timing delay $\Delta T$ (for a pulsar).

- Timing: Almost every systematic monitoring of the timing of signals from pulsars has the potential to detect planets around the pulsar. One pulsar (PSR1257+12) has revealed the presence of not one but three planets, with masses comparable to the Moon and Earth masses. Planetary systems around two other known pulsars need to be confirmed.

- Occultations: It is worthwhile to give some more details about the occultation method since it is the only technique with which we can hope to detect habitable planets in the near future. From the ground, the photometric precision is about $10^{-3}$, so it is only possible to use the method when the central star is roughly four-times smaller than the Sun (i.e., a dwarf star). It was originally argued that the tidal locking of the planet, which is very close to the star, excludes the presence of liquid water so the planet would not support life. However, it has been shown recently that three-dimensional atmospheric circulation may prevent the water from evaporating. A photometric monitoring of a few eclipsing binaries using multi-site campaigns (to have a time coverage as close as possible to 24 hours) started in 1994. Recently, one has perhaps seen in archived data of the $\beta$ Pic star an occultation by a Jupiter-like planet in 1980. It is impossible to

Observable quantities for Jupiter and Earth-like planets compared with instrument performance in 20 years ( ${ }^{*}$ for a parent star of 1 solar mass at $5 \mathrm{pc}$ from the Sun).

\begin{tabular}{llll}
\hline Parameter & Jupiter-like $^{*}$ & Earth-like $^{*}$ & Performance $^{-}$ \\
\hline Amplitude of star's wobbling & $80 \mu$ arcsec & $0.6 \mu$ arcsec & $20 \mu$ arcsec \\
Star-to-planet brightness ratio & $10^{-9}$ & $2 \times 10^{-10}$ & $\approx 10^{-9}$ \\
Star's velocity perturbation & $13 \mathrm{~m} / \mathrm{s}$ & $0.1 \mathrm{~m} / \mathrm{s}$ & $1 \mathrm{~m} / \mathrm{s}$ \\
Star's timing perturbation & $5 \mathrm{~s}$ & $3 \mathrm{~ms}$ & $\approx 5 \mu \mathrm{s}$ \\
Relative brightness decrease & $10^{-2}$ & $10^{-4}$ & $3 \times 10^{-5}$ \\
Relative brightness amplification & $\leq 0.3$ & $\approx 3 \times 10^{-2}$ & $\approx 10^{-2}$ \\
\hline
\end{tabular}

** For a planet $1 \mathrm{kpc}$ from the solar system confirm retrospectively this observation, but if the occultation is real, a second should be observed within the next decade. It is also necessary to start astrometric and spectroscopic monitoring of this star.

\section{New Campaigns Sought}

Many ideas and projects are mobilising several groups, mainly in the USA. An important improvement in spectroscopic performance should come within a few years when one expects a precision of $1 \mathrm{~m} / \mathrm{s}$. Two major ground-based projects in astrometry are very promising, but they are waiting for funding. One is based at the Keck telescope in Hawaii (the largest in the world with two $10 \mathrm{~m}$ mirrors) and the other at the European Very Large Telescope (VLT) which will consist of four $8 \mathrm{~m}$ mirrors in Chile (plus three additional $2 \mathrm{~m}$ mirrors for the VISA interferometric mode). In both cases, interferometry between the mirrors will be able to achieve a precision of 10 to 20 marcsec. NASA has a project to dedicate one-third of the Keck observing time to the search for planetary systems.

In space, where the photometric precision $\left(2 \times 10^{-5}\right)$ allows for searches around solartype stars, the aim is to monitor photometrically about one thousand stars. If all stars have terrestrial planets, one should then detect 20 of them. Two US projects are currently under study. In Europe, the European Space Agency (ESA) has the STARS satellite-based project while the COROT team of the French space agency CNES is examining the feasibility of a similar project. Both teams have recently agreed to include the search for occultations. An ESA ad hoc working group recently recommended a study of the feasibility of an astrometric mission. In its current version, named GAIA, it would also reach a precision of 10 to $20 \mu$ arcsec but on a significantly larger sample of stars.

\section{The Search for $\mathrm{O}_{2}$ and $\mathrm{O}_{3}$}

The final goal of detecting habitable planets is to detect signatures of life. One very promising approach is the detection of molecular abundances, compatible only with very out-of-equilibrium chemical reactions and similar to the high abundance of $\mathrm{O}_{2}$ in the Earth's atmosphere resulting from chlorophyllian photosynthesis. It has been suggested to search, by analogy, for $\mathrm{O}_{2}$ in the optical band at $760 \mathrm{~nm}$ and for $\mathrm{O}_{3}$ at $9.6 \mu \mathrm{m}$.

The $\mathrm{O}_{2}$ band can be sought either by spectro-imaging of the planet or by pure spectroscopy during a planetary transit. In the first case, a spatial mission would be necessary; in the second case is is worthwhile to investigate whether the observations could be made from the ground with a sufficiently large optical reflector (since a high angular resolution is unnecessary for spectroscopy). 
In the case of the $\mathrm{O}_{3}$ band, a satellite platform is required to avoid absorption by the Earth's atmosphere. A more careful investigation, such as for instance the DARWIN project submitted to the European Space Agency, shows that it is even necessary to go to $3.5 \mathrm{AU}$ or more from Earth to suppress the IR background of the zodiacal light.

The detection of planets around main sequence stars other than the Sun remains a major astrophysical and instrumental challenge. Today, one confirmed planetary system has been found, but at a place where almost nobody expected it (around a pulsar). There are also two possible candidates, but they need to be confirmed. Another intri- guing result is the lack of giant (with a mass equivalent to $\approx 2$ Jupiter masses) planets around the nearest bright stars.

Several ambitious programmes are in sight, and some of them will even be capable of detecting habitable planets and eventually signs of life. But perhaps more encouraging is to be able to assist in the awaking in Europe of a field that has the potential to greatly influence not only science but also our understanding of life.

Updates of this paper are accessible on the World-Wide Web in the Extrasolar Planets Encyclopedy as http://mesiop.obspm.fr/ schneider/plan/encycl.html

\section{POSITRON MICROSCOPY}

\section{Pulse Microbeam in Operation}

The primary beam of a positron scanning microscope based on using the positron lifetime technique to detect defects such as vacancies or voids [see EN 25 (1994) 178]

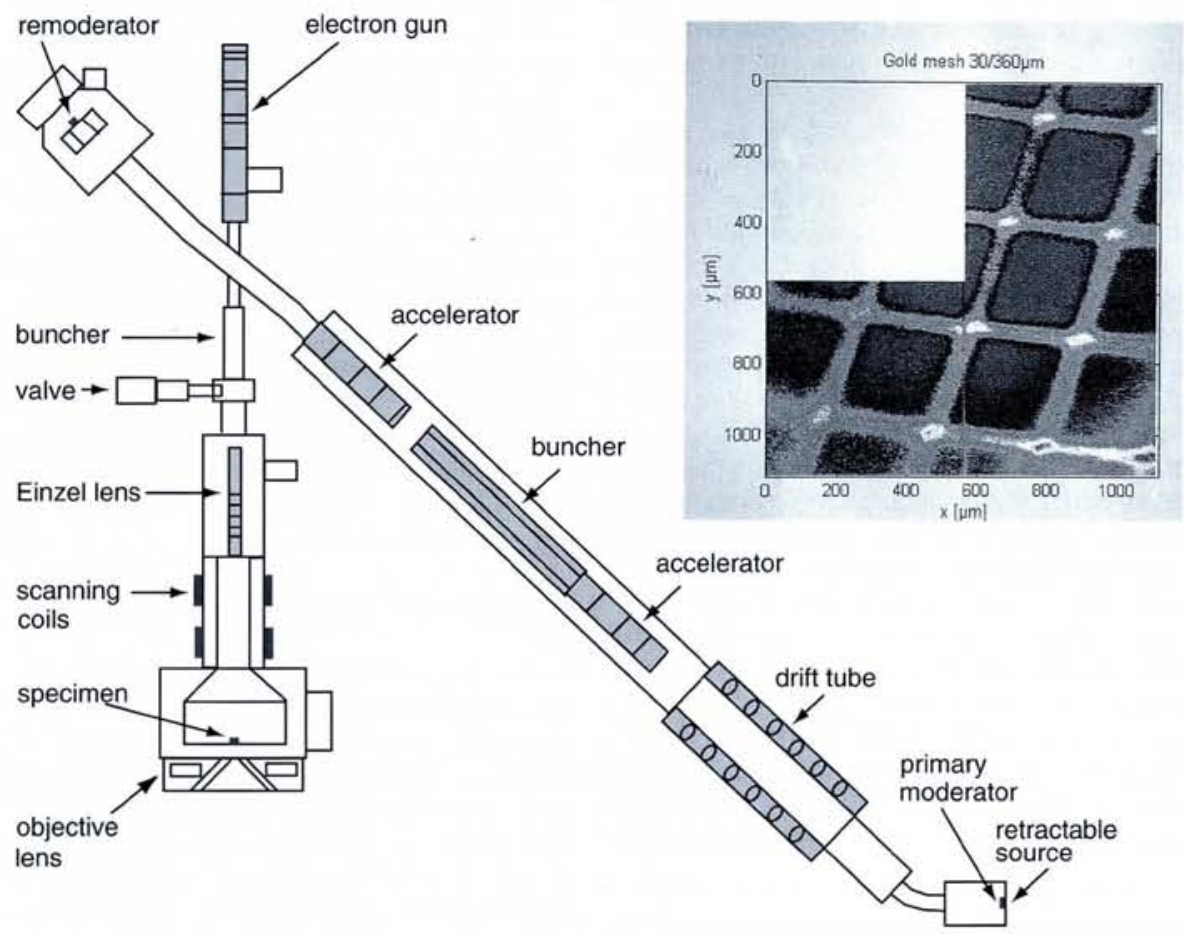

Schematic layout of the scanning positron microscope which aims to produce a few microns spot size. The project is being carried out at Trento University and at the Universität der Bundeswehr in Munich under a European Union BRITE-EURAM grant. The inclined pulsed beam system on the right is integrated with a specially designed electron microscope shown on the left. It demagnifies the spot to about $20 \mu \mathrm{m}$ and generates 300 ps pulses. The electrooptical column will reduce the spot diameter down to a few $\mathrm{mm}$ and the pulse duration to below 150 ps. It will also scan the beam over the sample and control the beam energy. The novel design of the primary beam system allows a high transport efficiency even with the losses owing to beam pulsing (the $1 \mathrm{mCi}{ }^{22} \mathrm{Na}$ source yields 5000 positrons per second in the $20 \mu \mathrm{m}$ spot). This implies that easy to handle, relatively cheap positron sources can be used. Inset is the contrast image of a gold grid ( $360 \mu \mathrm{m}$ mesh size; $30 \mu \mathrm{m}$ wire diameter) generated by scanning the primary beam. beam across a gold grid placed at the image position of the last lens. The image (see figure) is not yet a lifetime image, but a contrast image which allows one to estimate the spatial resolution as being better than $20 \mu \mathrm{m}$. It compares very favourably with the few images produced in the last years using positron beams. The novelty is that it has been obtained with a pulsed beam. The time resolution achieved with the primary column is close to $350 \mathrm{ps}$. Planned refinements should allow a pulse duration of the order of $150 \mathrm{ps}$ to be reached. The beam will then simultaneously exhibit a spot size and a time resolution close to the best values achieved until now in separate systems. The energy of the positron beam will be variable from 1 to $30 \mathrm{keV}$. In this way the positron implantation profile can be varied and a non-destructive depth profiling of the sample made possible.

The lifetime method has been used over the last 25 years with positrons from radioactive sources (depth resolution of the order of $100 \mu \mathrm{m}$; lateral resolution of the order of a few millimetres). More recently, it has been used with pulsed positron beams of a few $\mathrm{mm}$ in diameter. In both cases, the possibility of distinguishing up to four types of defects has been demonstrated; the defect concentration can be obtained from the intensity of a given lifetime component. The positron microscope will perform the same kind of measurements on a pixel size of the order of a few square microns. Scanning the beam over the sample surface will produce a two-dimensional defect image and energy scanning will allow the generation of threedimensional images with a variable depth resolution (of the order of $10 \mathrm{~nm}$ at depth of $100 \mathrm{~nm}$, increasing to some $500 \mathrm{~nm}$ at a $2 \mu \mathrm{m})$.

The other type of positron microscope is based on re-emission. A beam of moderated positrons from a ${ }^{22} \mathrm{Na}$ source is focussed on the sample and the untrapped, re-emitted positron intensity is magnified and then imaged using a two-dimensional positronsensitive detector. A $10^{4}$-fold magnification of the re-emitted positron distribution has been achieved. Prototypes based on this approach, which is is limited to metals that re-emit positrons and to the study of surface features that affect positron emission (as opposed to near-surface structures), have been constructed in the US (Brandeis) and the UK (East Anglia).

A. Zecca, Trento

\section{Corrigendum}

The following sentence should be added at the end of the article "Positron Annihilation: Industrial Applications DeveIop", published in Europhysics News 25 (1994) 178: "This workshop has been made possible through the financial support of the Royal Netherlands Academy of Sciences and of the European Commission's Directorate-General XII». Furthermore, in the last sentence of the figure caption on p. 179, the word "delocalization" should read "Iocalization". 